PROCEEDINGS OF THE

AMERICAN MATHEMATICAL SOCIETY

Volume 130, Number 7, Pages 2115-2123

S 0002-9939(01)06308-0

Article electronically published on December 20, 2001

\title{
SOME VARIATIONAL FORMULAS ON ADDITIVE FUNCTIONALS OF SYMMETRIC MARKOV CHAINS
}

\author{
DAEHONG KIM, MASAYOSHI TAKEDA, AND JIANGANG YING \\ (Communicated by Claudia M. Neuhauser)
}

\begin{abstract}
For symmetric continuous time Markov chains, we obtain some formulas on total occupation times and limit theorems of additive functionals by using large deviation theory.
\end{abstract}

\section{INTRODUCTION}

In [11, we extended Donsker-Varadhan's type large deviation of symmetric Markov processes with finite lifetime and established the full large deviation principle; let $\mathbf{M}=\left(P_{x}, X_{t}, \zeta\right)$ be an $m$-symmetric Markov process on a locally compact separable metric space $X$. Here $\zeta$ is the lifetime of $\mathbf{M}$ and $m$ is a Radon measure with full support. If the process $\mathbf{M}$ explodes rapidly in the sense that the 1-resolvent of the identity function $1, R_{1} 1(x)\left(=1-E_{x}(\exp (-\zeta))\right)$, belongs to $C_{\infty}(X)$, the space of continuous functions vanishing at infinity, then the full large deviation principle is derived (Theorem 3.1 and Theorem 4.4 in [11]). As a corollary, the following variational formula on the lifetime $\zeta$ is obtained:

$$
\lim _{t \rightarrow \infty} \frac{1}{t} \log P_{x}(t<\zeta)=-\inf \left\{\mathcal{E}(u, u): u \in \mathcal{F}, \int_{X} u^{2} d m=1\right\}
$$

for any $x \in X$, where $(\mathcal{E}, \mathcal{F})$ is the Dirichlet form on $L^{2}(X ; m)$ generated by $\mathbf{M}$. As an application of formula (1), we considered in [12 tail probabilies of $A_{\tau_{D}}$, where $A_{t}$ is a positive continuous additive functional of the Brownian motion and $\tau_{D}$ is the exit time from a regular domain $D$.

In this paper, we shall treat the same problem for symmetric continuous time Markov chains. Our results are also formulated as an application of formula (1) but contain the accurate calculations of its exponential decay rates represented by Dirichlet forms. Let $I$ be a countable set with the discrete topology. Let $\mathcal{Q}=\left(q_{i j}\right)$ be an $I \times I$ matrix such that $m_{i} q_{i j}=m_{j} q_{j i}$ for some strictly positive function $m_{i}$ on $I$. Denote by $\mathbf{M}=\left(P_{i}, X_{t}\right)$ the minimal $\mathcal{Q}$-process with lifetime $\zeta, \zeta=\inf \{t>$ $\left.0: X_{t}=\Delta\right\}$. Here $\Delta$ is the one-point compactification of $I$. We shall show our main theorem in section 2 .

Received by the editors May 20, 2000 and, in revised form, January 29, 2001.

2000 Mathematics Subject Classification. Primary 60F10, 60J20; Secondary 31C25.

Key words and phrases. Additive functional, Dirichlet form, large deviation, symmetric Markov chain.

The first author's research was supported in part by Brain Korea 21.

(C)2001 American Mathematical Society 
Theorem 1.1. Let $\mu$ be a non-negative function on I such that

$$
\lim _{i \rightarrow \Delta} E_{i}\left(\exp \left(-\int_{0}^{\zeta} \frac{\mu}{m}\left(X_{t}\right) d t\right)\right)=1
$$

We have for any $i \in I$,

$$
\begin{aligned}
\lim _{\beta \rightarrow \infty} & \frac{1}{\beta} \log P_{i}\left(\int_{0}^{\zeta} \frac{\mu}{m}\left(X_{t}\right) d t>\beta\right) \\
= & -\inf \left\{\mathcal{E}(u, u): u \in \mathcal{F}, \sum_{i \in I} u^{2}(i) \mu_{i}=1\right\},
\end{aligned}
$$

where $(\mathcal{E}, \mathcal{F})$ is the Dirichlet form associated with the Markov chain $\mathbf{M}$.

In section 3, we shall consider concrete examples as an application of Theorem 1.1, and extend D. Freedman's theorem on stochastic interpretation of $q_{i j}$ in our setting.

Let us consider the discontinuous additive functional of the form

$$
A_{t}^{\mu, F}=A_{t}^{\mu}+\sum_{0<s \leq t} F\left(X_{s-}, X_{s}\right)
$$

where $A_{t}^{\mu}$ is the positive continuous additive functional of the form (5) below for a non-negative function $\mu$ on $I$. Under certain conditions on $\mathbf{M}$ and $F$, we can see from [7] that

$$
\lim _{t \rightarrow \infty} \frac{1}{t} \log E_{i}\left(\exp \left(A_{t}^{\mu, F}\right)\right)=-\inf \left\{\mathcal{E}^{\mu, F}(u, u): \sum_{i \in I} u^{2}(i) m_{i}=1\right\},
$$

where $\mathcal{E}^{\mu, F}$ is the symmetric form on $L^{2}(I ; m)$ generated by the semigroup

$$
p_{t}^{\mu, F} f(i)=E_{i}\left(\exp \left(A_{t}^{\mu, F}\right) f\left(X_{t}\right)\right) .
$$

In section 4 , we obtain the large deviation principle for $A_{t}^{\mu, F} / t$ by combining (3) with the Gärtner and Ellis theorem ([3]). In particular, we shall show that

$$
\frac{A_{t}^{\mu, F}}{t} \text { exponentially converges to } \frac{\mu(I)+\sum_{i \neq j} F(i, j) q_{i j} m_{i}}{m(I)} \text {. }
$$

\section{Proof of the main theorem}

Let $I$ be a countable set with the discrete topology. Let $\mathcal{Q}=\left(q_{i j}\right)$ be an $I \times I$ matrix such that

$$
q_{i j} \geq 0 \quad(i \neq j), \quad \sum_{k \neq i} q_{i k} \leq-q_{i i}<\infty, \quad \forall i \in I
$$

and $m_{i} q_{i j}=m_{j} q_{j i}$ for some strictly positive function $m_{i}$ on $I$. Let $\mathcal{E}$ be the Dirichlet form on $L^{2}(I ; m)$ defined by

$$
\mathcal{E}(u, v)=\frac{1}{2} \sum_{i \neq j}(u(j)-u(i))(v(j)-v(i)) q_{i j} m_{i}+\sum_{i} u(i) v(i)\left(-q_{i i}-\sum_{j \neq i} q_{i j}\right) m_{i} .
$$


Denote by $\mathcal{F}^{r}$ the collection of functions $u$ on $I$ such that $\mathcal{E}(u, u)<\infty$. Let $\mathcal{F}$ be the set of $L^{2}(I ; m)$-functions $u$ in $\mathcal{F}^{r}$ for which there exists $u_{n}(n=1,2, \ldots$,$) with$ finite support such that

$$
u_{n} \rightarrow u \quad \text { and } \sup _{n} \mathcal{E}\left(u_{n}, u_{n}\right)<\infty .
$$

Then $(\mathcal{E}, \mathcal{F})$ becomes a regular Dirichlet form on $L^{2}(I ; m)$. Denote by $\mathbf{M}=$ $\left(\Omega, X_{t}, P_{i}, \zeta\right)$ the Hunt process generated by $(\mathcal{E}, \mathcal{F})$, which is nothing but the minimal $\mathcal{Q}$-process constructed by W. Feller (Theorem 17.2 in $[10]$ ). Here $\Omega$ is the space of all right continuous maps of $[0, \infty)$ into $I \cup\{\Delta\}$, the one-point compactification of $I$. When $I$ is finite, $\Delta$ is regarded as an isolated point. $\zeta$ is the lifetime of $\mathbf{M}$, $\zeta=\inf \left\{t>0: X_{t}=\Delta\right\}$. Let us denote by $\left\{p_{t}\right\}_{t \geq 0}$ and $\left\{R_{\alpha}\right\}_{\alpha>0}$ the semigroup and resolvent of $\mathbf{M}$ respectively, i.e., $p_{t} f(i)=E_{i}\left(f\left(X_{t}\right)\right), R_{\alpha} f(i)=E_{i}\left(\int_{0}^{\zeta} e^{-\alpha t} f\left(X_{t}\right) d t\right)$. We then make following assumption:

(A) (Irreducibility of M.) For any $i, j \in I, P_{i}\left(\sigma_{j}<\zeta\right)>0$, where $\sigma_{j}$ is the first hitting time of $j, \sigma_{j}=\inf \left\{t>0: X_{t}=j\right\}$.

Let $\mathcal{B}^{+}$be the set of all non-negative functions on $I$. We introduce the subspace $\mathcal{K}$ of $\mathcal{B}^{+}$,

$$
\mathcal{K}=\left\{\mu \in \mathcal{B}^{+}: \lim _{i \rightarrow \Delta} E_{i}\left(\exp \left(-A_{\zeta}^{\mu}\right)\right)=1\right\}
$$

where $A_{t}^{\mu}$ is the positive continuous additive functional of the form

$$
A_{t}^{\mu}=\int_{0}^{t} \frac{\mu}{m}\left(X_{s}\right) d s .
$$

Let us denote by $\tau_{t}(t \geq 0)$ the right continuous inverse of $A_{t}^{\mu}$,

$$
\tau_{t}=\inf \left\{s>0: A_{s}^{\mu}>t\right\} .
$$

The time changed process $Y_{t}^{\mu}$ of $X_{t}$ with respect to $A_{t}^{\mu}$ is defined by

$$
Y_{t}^{\mu}=X_{\tau_{t}} \text {. }
$$

Let $F=\left\{i \in I: \mu_{i}>0\right\}$. The process $Y_{t}^{\mu}$ is then a $\mu$-symmetric Hunt process on $F$ with lifetime $A_{\zeta}^{\mu}$ (Theorem 6.2.3 in [6] and Theorem 65.9 in [9]). Set

$$
H_{F} u(i)=E_{i}\left(u\left(X_{\sigma_{F}}\right): \sigma_{F}<\zeta\right),
$$

where $\sigma_{F}=\inf \left\{t>0: X_{t} \in F\right\}$. Then the time changed process $Y_{t}^{\mu}$ of $X_{t}$ generates the following Dirichlet form $(\check{\mathcal{E}}, \check{\mathcal{F}})$ on $L^{2}(F ; \mu)$ :

$$
\left\{\begin{array}{l}
\check{\mathcal{F}}=\left\{\varphi \in L^{2}(F ; \mu): \varphi=u \text { on } F \text { for some } u \in \mathcal{F}\right\} \\
\check{\mathcal{E}}(\varphi, \varphi)=\mathcal{E}\left(H_{F} u, H_{F} u\right), \varphi \in \check{\mathcal{F}}, \varphi=u \text { on } F u \in \mathcal{F}_{e} .
\end{array}\right.
$$

Here $\mathcal{F}_{e}$ stands for the extended Dirichlet space of $(\mathcal{E}, \mathcal{F})$ (Theorem 6.2.1 in [6]).

Let $\left\{G_{\alpha}^{\mu}(i, j)\right\}_{\alpha \geq 0}$ be the resolvent kernel of $Y_{t}^{\mu}$,

$$
G_{\alpha}^{\mu}(i, j)=E_{i}\left(\int_{0}^{\infty} e^{-\alpha t} I_{\{j\}}\left(Y_{t}^{\mu}\right) d t\right) .
$$

Then by the definition of the time changed process and the fact that $A_{\zeta}^{\mu}$ is the lifetime of $Y_{t}^{\mu}$, we have the following:

$$
G_{0}^{\mu}(i, j)=E_{i}\left(\int_{0}^{\zeta} I_{\{j\}}\left(X_{t}\right) d t\right) \frac{\mu_{j}}{m_{j}} \quad \text { for } i, j \in F
$$


and

$$
E_{i}\left(\int_{0}^{\infty} e^{-t} I_{F}\left(Y_{t}^{\mu}\right) d t\right)=E_{i}\left(\int_{0}^{A_{\zeta}^{\mu}} e^{-t} d t\right)=1-E_{i}\left(\exp \left(-A_{\zeta}^{\mu}\right)\right) .
$$

Since $G_{0}^{\mu}(i, j)>0$ for any $i, j \in F$ due to (7), the process $Y_{t}^{\mu}$ is irreducible. Moreover, equation (8) says that for $\mu \in \mathcal{K}$, the 1-resolvent $G_{1}^{\mu}$ of the identity function $1_{F}$ vanishes at infinity $\Delta$. Therefore, we obtain Theorem 1.1 by exactly the same argument as Theorem 3.1 in [12].

Remark 2.1. (i) We can easily see that the statement of Theorem 4.1 in [11] still holds even if $P_{x}$ is replaced by $\sup _{x \in X} P_{x}$. As a result, we see that

$$
\lim _{\beta \rightarrow \infty} \frac{1}{\beta} \log \sup _{i \in I} P_{i}\left(A_{\zeta}^{\mu}>\beta\right) \leq-\inf \left\{\mathcal{E}(u, u): u \in \mathcal{F}, \sum_{i \in I} u^{2}(i) \mu_{i}=1\right\} .
$$

(ii) For $\mu \in \mathcal{B}^{+}$, assume that the support of $\mu$ is a finite set. Then $\mu$ belongs to the class $\mathcal{K}$.

(iii) If $\lim _{i \rightarrow \Delta} E_{i}\left(A_{\zeta}^{\mu}\right)=0$, then $\mu$ belongs to the class $\mathcal{K}$.

\section{EXAMPLES}

For a Borel subset $B$ of $I \times I$, let $J_{B}$ be the terminal time defined by

$$
J_{B}=\inf \left\{t>0:\left(X_{t-}, X_{t}\right) \in B\right\}
$$

and $X_{t}^{B}$ the killed process defined by

$$
X_{t}^{B}=\left\{\begin{array}{cl}
X_{t} & \text { on } t<J_{B}, \\
\Delta & \text { on } t \geq J_{B}
\end{array} \quad\left(X_{\infty}^{B}=\Delta\right)\right.
$$

The process $X_{t}^{B}$ is then a Hunt process on the state space

$$
I^{B}=\left\{x \in I: P_{x}\left(J_{B}>0\right)=1\right\} .
$$

Note that $X_{t}^{B}$ is not always symmetric; however, we see from Theorem 3.10 in [13] that it is nearly symmetric. More precisely, let us define the bilinear form $\left(\mathcal{E}^{B}, \mathcal{F}^{B}\right)$ on $L^{2}\left(I^{B} ; m\right)$ by

$$
\left\{\begin{array}{l}
\mathcal{E}^{B}(u, v)=\mathcal{E}(u, v)+\sum_{(i, j) \in B} u(i) v(j) q_{i j} m_{i}, \\
\mathcal{F}^{B}=\mathcal{F} \cap\left\{u: \sum_{(i, j) \in B} u^{2}(i) q_{i j} m_{i}<\infty, \sum_{(i, j) \in B} u^{2}(j) q_{i j} m_{i}<\infty\right\} .
\end{array}\right.
$$

Then, $\left(\mathcal{E}^{B}, \mathcal{F}^{B}\right)$ is the non-symmetric Dirichlet form generated by the process $X_{t}^{B}$.

Let $K$ be a finite subset of $I^{B}$ and consider the time changed process $Y_{t}^{K}$ of $X_{t}^{B}$ with respect to $\int_{0}^{t} I_{K}\left(X_{s}\right) d s$,

$$
Y_{t}^{K}=X_{\tau_{t}}^{B}, \quad \tau_{t}=\inf \left\{s>0: \int_{0}^{s} I_{K}\left(X_{u}\right) d u>t\right\} .
$$

Then, $Y_{t}^{K}$ is a Hunt process on $K$ with lifetime $\zeta=\int_{0}^{J_{B}} I_{K}\left(X_{s}\right) d s$. Set $H_{K} u(j)=$ $E_{j}\left(u\left(X_{\sigma_{K}}\right): \sigma_{K}<J_{B}\right)$, where $\sigma_{K}=\inf \left\{t>0: X_{t} \in K\right\}$. The Dirichlet form $\left(\mathcal{E}^{B}, \check{\mathcal{F}}^{B}\right)$ on $L^{2}(K ; m)$ generated by the time changed process $Y_{t}^{K}$ is as follows:

$$
\left\{\begin{array}{l}
\check{\mathcal{F}}^{B}=\left\{\varphi \in L^{2}(K ; m): \varphi=u \text { on } K \text { for some } u \in \mathcal{F}_{e}^{B}\right\} \\
\check{\mathcal{E}}^{B}(\varphi, \varphi)=\mathcal{E}^{B}\left(H_{K} u, H_{K} u\right), \varphi \in \check{\mathcal{F}}^{B}, \varphi=u \text { on } K u \in \mathcal{F}_{e}^{B} .
\end{array}\right.
$$


Here $\mathcal{F}_{e}^{B}$ stands for the extended Dirichlet space of $\left(\mathcal{E}^{B}, \mathcal{F}^{B}\right)$. Now by applying Theorem 1.1 and Remark 2.2 (ii) to $X_{t}^{B}$, we obtain the asymptotic formula for the total occupation times as follows:

Proposition 3.1. Assume that $X_{t}^{B}$ is irreducible and that $Y_{t}^{K}$ is symmetric with respect to $m$. Then, for any $i \in I^{B}$

$$
\begin{aligned}
\lim _{\beta \rightarrow \infty} & \frac{1}{\beta} \log P_{i}\left(\int_{0}^{J_{B}} I_{K}\left(X_{s}\right) d s>\beta\right) \\
= & -\inf \left\{\check{\mathcal{E}^{B}}(u, u): u \in \check{\mathcal{F}^{B}}, \sum_{i \in K} u^{2}(i) m_{i}=1\right\} .
\end{aligned}
$$

Now, let us consider some concrete examples of (9), for which we mainly concentrate on the calculations of exponential decay rates represented by Dirichlet forms.

Example 3.1. Let $B=I \times D(D \subset I)$ and $K \subset I \backslash D$. Then, $J_{B}$ is the hitting time $\sigma_{D}$ of $D, \sigma_{D}=\inf \left\{t>0: X_{t} \in D\right\}$, and $H_{K} u(j)=E_{j}\left(u\left(X_{\sigma_{K}}\right): \sigma_{K}<\sigma_{D}\right)$. If $X_{t}^{B}$ is irreducible, then the assumptions in Proposition 3.1 are fulfilled. Hence, we have

$$
\begin{aligned}
\lim _{\beta \rightarrow \infty} \frac{1}{\beta} \log P_{j}\left(\int_{0}^{\sigma_{D}} I_{K}\left(X_{s}\right) d s>\beta\right) \\
=-\inf \left\{\mathcal{E}^{B}\left(H_{K} u, H_{K} u\right): \sum_{i \in K} u^{2}(i) m_{i}=1\right\}
\end{aligned}
$$

for any $j \in I \backslash D$. In particular, let $K$ be a one point set $\{a\}$. Then, since

$$
H_{\{a\}} u(j)=E_{j}\left(u\left(X_{\sigma_{\{a\}}}\right): \sigma_{\{a\}}<\sigma_{D}\right)=u(a) P_{j}\left(\sigma_{\{a\}}<\sigma_{D}\right)
$$

and $\operatorname{Cap}^{I \backslash D}(\{a\})=\mathcal{E}^{B}\left(P_{j}\left(\sigma_{\{a\}}<\sigma_{D}\right), P_{j}\left(\sigma_{\{a\}}<\sigma_{D}\right)\right)$ on account of Theorem 4.3.3 in [6], we obtain

$$
\begin{aligned}
& \lim _{\beta \rightarrow \infty} \frac{1}{\beta} \log P_{j}\left(\int_{0}^{\sigma_{D}} I_{\{a\}}\left(X_{s}\right) d s>\beta\right) \\
& =-\inf \left\{\mathcal{E}^{B}\left(H_{\{a\}} u, H_{\{a\}} u\right): u^{2}(a) m_{a}=1\right\} \\
& =-\frac{\operatorname{Cap}^{I \backslash D}(\{a\})}{m_{a}},
\end{aligned}
$$

where $\operatorname{Cap}^{I \backslash D}(\{a\})$ is the 0 -order capacity defined by $\operatorname{Cap}^{I \backslash D}(\{a\})=\inf \left\{\mathcal{E}^{B}(u, u)\right.$ : $u \in \mathcal{F}, u(a)=1, u(i)=0 \forall i \in D\}(\underline{6})$. Indeed, let $G(i, j)$ be the green function of the part process on $I \backslash D$ and $F(a)=P_{a}\left(\hat{\sigma}_{\{a\}}<\infty\right), \sigma_{\{a\}}=\inf \left\{t>\sigma_{I \backslash\{a\}}\right.$ : $\left.X_{t}=a\right\}$. Denote by $R(a)$ the expected number of visits to $a$ with respect to $P_{a}$. Then, since

$$
R(a)=1+F(a)+F^{2}(a)+\cdots=\frac{1}{1-F(a)}, \quad G(a, a) m_{a}=\frac{R(a)}{q_{a a}},
$$

and

we see that

$$
\operatorname{Cap}^{I \backslash D}(\{a\})=\frac{1}{G(a, a)},
$$

$$
\frac{\operatorname{Cap}^{I \backslash D}(\{a\})}{m_{a}}=q_{a a}(1-F(a)) .
$$


Hence, equation (11) can also be derived from Proposition (5.3) in [1].

Example 3.2. Let $B=\{a\} \times D(D \subset I \backslash\{a\})$. Then

$$
\mathcal{E}^{B}(u, v)=\mathcal{E}(u, v)+\sum_{j \in D} u(a) v(j) q_{a j} m_{a} .
$$

Assume that $X_{t}^{B}$ is irreducible and $K$ is a one point set $\{a\}$. We then have

$$
\begin{aligned}
& \lim _{\beta \rightarrow \infty} \frac{1}{\beta} \log P_{j}\left(\int_{0}^{J_{B}} I_{\{a\}}\left(X_{s}\right) d s>\beta\right) \\
& =-\inf \left\{\mathcal{E}^{B}\left(H_{\{a\}} u, H_{\{a\}} u\right): u^{2}(a) m_{a}=1\right\}
\end{aligned}
$$

for any $j \in I^{B}$. By again noting $H_{\{a\}} u(j)=E_{j}\left(u\left(X_{\sigma_{\{a\}}}\right): \sigma_{\{a\}}<J_{B}\right)=$ $u(a) P_{j}\left(\sigma_{\{a\}}<\zeta\right)$, we see that the right-hand side of (12) is equal to

$$
\begin{aligned}
& u^{2}(a) \mathcal{E}\left(P_{j}\left(\sigma_{\{a\}}<\zeta\right), P_{j}\left(\sigma_{\{a\}}<\zeta\right)\right)+\sum_{j \in D} u^{2}(a) q_{a j} m_{a} P_{j}\left(\sigma_{\{a\}}<\zeta\right) \\
& =\frac{\operatorname{Cap}(\{a\})}{m_{a}}+\sum_{j \in D} q_{a j} P_{j}\left(\sigma_{\{a\}}<\infty\right) .
\end{aligned}
$$

Here Cap is 0-order capacity associated with the Dirichlet form $(\mathcal{E}, \mathcal{F})$. If $X_{t}$ is recurrent, then $\operatorname{Cap}(\{a\})=0$ and $P_{j}\left(\sigma_{\{a\}}<\infty\right)=1$. Moreover, since $\int_{0}^{J_{B}} I_{\{a\}}\left(X_{s}\right) d s$ is a random variable with the exponential distribution with respect to $P_{a}$, we see that $\int_{0}^{J_{B}} I_{\{a\}}\left(X_{s}\right) d s$ has the exponential distribution of parameter

$$
\sum_{j \in D} q_{a j}
$$

As a result, we rediscover D. Freedman's probabilistic interpretation of the component $q_{i j}$ (Theorem (2) in [5]) through the large deviation theory.

Example 3.3. Let $I=\{0,1,2, \ldots\}$ and $X$ be a birth and death process with birth rate $\phi_{i}$ and death rate $\varphi_{i}$ on $I$. We denote by

$$
s_{0}=0, \quad s_{1}=\frac{1}{\phi_{0}}, \quad s_{n}=\frac{1}{\phi_{0}}+\sum_{i=1}^{n-1} \frac{1}{\phi_{i} m_{i}}
$$

where

$$
m_{0}=1, \quad m_{i}=\frac{\phi_{0} \phi_{1} \cdots \phi_{i-1}}{\varphi_{1} \varphi_{2} \cdots \varphi_{i}}, \quad i=1,2, \ldots
$$

Then the corresponding Dirichlet form on $L^{2}(I ; m)$ of $X$ is as follows:

$$
\begin{aligned}
\mathcal{E}(u, v) & =\sum_{i=0}^{\infty}\left(\frac{u(i+1)-u(i)}{s_{i+1}-s_{i}}\right)\left(\frac{v(i+1)-v(i)}{s_{i+1}-s_{i}}\right)\left(s_{i+1}-s_{i}\right) \\
& =\sum_{i=0}^{\infty}(u(i+1)-u(i))(v(i+1)-v(i)) \phi_{i} m_{i}, \quad u, v \in \mathcal{F} .
\end{aligned}
$$

Let $B=I \times\{n\}$ and $K=\{a, a+1, \ldots, a+k-1\}(0 \leq a<a+k-1<n)$. Then the terminal time $J_{B}$ is the hitting time $\sigma_{\{n\}}$ of $\{n\}$. By observing $P_{i}\left(\sigma_{K}<\sigma_{\{n\}}\right)=1$ for $0 \leq i \leq a+k-1$ and

$$
P_{i}\left(\sigma_{K}<\sigma_{\{n\}}\right)=\frac{s_{n}-s_{i}}{s_{n}-s_{a+k-1}} \quad \text { for } \quad a+k \leq i<n,
$$


we have

$$
\mathcal{E}^{B}\left(H_{K} u, H_{K} u\right)=\sum_{i=a}^{a+k-2}(u(i+1)-u(i))^{2} \phi_{i} m_{i}+\frac{u^{2}(a+k-1)}{s_{n}-s_{a+k-1}} .
$$

Consequently, the right-hand side of (9) is the extremum problem of (14) with condition

$$
\sum_{i=a}^{a+k-1} u^{2}(i) m_{i}=1
$$

For convenience, we put $u(a+i)=x_{i}, i=0,1, \ldots, k-1$,

$$
G\left(x_{0}, \ldots, x_{k-1}\right)=\sum_{i=1}^{k-1}\left(x_{i}-x_{i-1}\right)^{2} \phi_{i} m_{i}+\frac{x_{k-1}^{2}}{s_{n}-s_{a+k-1}}
$$

and

$$
F\left(\lambda, x_{0}, \ldots, x_{k-1}\right)=G\left(x_{0}, \ldots, x_{k-1}\right)+\lambda\left(\sum_{i=0}^{k-1} x_{i}^{2} m_{i}-1\right) .
$$

Then by (15)

$$
\frac{x_{0}}{2} \frac{\partial F}{\partial x_{0}}+\frac{x_{1}}{2} \frac{\partial F}{\partial x_{1}}+\cdots+\frac{x_{k-1}}{2} \frac{\partial F}{\partial x_{k-1}}=G\left(x_{0}, \ldots, x_{k-1}\right)+\lambda=0 .
$$

Hence we conclude that

$$
\begin{aligned}
\lim _{\beta \rightarrow \infty} \frac{1}{\beta} \log P_{i}\left(\int_{0}^{\sigma_{\{n\}}} I_{K}\left(X_{s}\right) d s>\beta\right) \\
=-\inf \left\{\mathcal{E}\left(H_{K} u, H_{K} u\right): \sum_{i \in K} u^{2}(i) m_{i}=1\right\} \\
=\text { the maximum solution of } \operatorname{det}(\boldsymbol{A})=0,
\end{aligned}
$$

where

$$
\begin{gathered}
\boldsymbol{A}=\left(\begin{array}{ccccc}
U_{a} & -V_{a} & 0 & \cdots & 0 \\
-V_{a} & V_{a}+U_{a+1} & -V_{a+1} & \cdots & 0 \\
0 & -V_{a+1} & V_{a+1}+U_{a+2} & \vdots & \vdots \\
\vdots & \vdots & \vdots & \vdots & \vdots \\
\ldots & \ldots & 0 & -V_{a+k-2} & \frac{1}{S_{n}-S_{a+k-1}}+U_{a+k-1}
\end{array}\right), \\
U_{i}=\phi_{i} m_{i}+\lambda m_{i} \text { and } V_{i}=\phi_{i} m_{i}, i=a, \ldots, a+k-1 .
\end{gathered}
$$

\section{The Large Deviation PRinciple FOR ADDitive FunCtionals}

In this section, we further assume that the state space $I$ is finite and the lifetime of $\mathbf{M}$ is infinity, $P_{i}(\zeta<\infty)=0$. Let us consider the discontinuous additive functional

$$
A_{t}^{\mu, F}=A_{t}^{\mu}+\sum_{0<s \leq t} F\left(X_{s-}, X_{s}\right)
$$


where $F$ is a Borel function on $I \times I$ such that $F(i, j)=F(j, i)$ (symmetry), $F(i, i)=0$. Then the symmetric bilinear form $\mathcal{E}^{\mu, F}$ on $L^{2}(I ; m)$ generated by the semigroup

can be written as

$$
p_{t}^{\mu, F} f(i)=E_{i}\left(\exp \left(A_{t}^{\mu, F}\right) f\left(X_{t}\right)\right)
$$

$$
\begin{aligned}
\mathcal{E}^{\mu, F}(u, v)= & \frac{1}{2} \sum_{i \neq j}(u(j)-u(i))(v(j)-v(i)) q_{i j} m_{i} \\
& -\sum_{i} u(i) v(i) \mu_{i}-\sum_{i \neq j} u(i) v(j)\left(1-e^{F(i, j)}\right) q_{i j} m_{i},
\end{aligned}
$$

and we have from [7]

$$
\lim _{t \rightarrow \infty} \frac{1}{t} \log E_{i}\left(\exp \left(\theta A_{t}^{\mu, F}\right)\right)=C(\theta) \quad \text { for all } i \in I,
$$

where

$$
C(\theta)=-\inf \left\{\mathcal{E}^{\theta \mu, \theta F}(u, u): \sum_{i \in I} u^{2}(i) m_{i}=1\right\} .
$$

Let us denote by $u_{\theta}$ the function attaining the infimum of the right-hand side above, that is, the first eigenfunction with eigenvalue $-C(\theta)$. Now, let us express $\mathcal{E}^{\theta \mu, \theta F}$ as a power series

$$
\mathcal{E}^{\theta \mu, \theta F}(u, u)=\mathcal{E}(u, u)+\theta \mathcal{E}^{(1)}(u, u)+\theta^{2} \mathcal{E}^{(2)}(u, u)+\cdots .
$$

Then $\mathcal{E}^{(1)}$ is written as

$$
\begin{aligned}
\mathcal{E}^{(1)}(u, u)= & \sum_{i \in I} u^{2}(i) \mu_{i}-\sum_{i \neq j}(u(j)-u(i))^{2} F(i, j) q_{i j} m_{i} \\
& +\sum_{i} u^{2}(i)\left(\sum_{j} F(i, j) q_{i j} m_{i}\right) .
\end{aligned}
$$

We see from 8 that $C(\theta)$ is differentiable with respect to $\theta$ and $C^{\prime}(\theta)=\mathcal{E}^{(1)}\left(u_{\theta}, u_{\theta}\right)$. In particular, since $u_{0}=1 / \sqrt{m(I)}$ (constant), we see that

$$
C^{\prime}(0)=\frac{\mu(I)+\sum_{i \neq j} F(i, j) q_{i j} m_{i}}{m(I)} .
$$

The Gärtner and Ellis theorem ([3]) tells us that $A_{t}^{\mu, F} / t$ obeys the large deviation principle with rate function $I(\lambda)(=\sup \{\lambda \theta-C(\theta)\})$; for any Borel set $\Lambda$ of $R^{1}$,

$$
-I\left(\Lambda^{\circ}\right) \leq \liminf _{t \rightarrow \infty} \frac{1}{t} \log -P_{i}\left(\frac{A_{t}^{\mu, F}}{t} \in \Lambda\right) \leq \limsup _{t \rightarrow \infty} \frac{1}{t} \log P_{i}\left(\frac{A_{t}^{\mu, F}}{t} \in \Lambda\right) \leq-I(\bar{\Lambda}),
$$

where $\Lambda^{\circ}$ and $\bar{\Lambda}$ are the interior and closure of $\Lambda$ respectively, and $I(\Lambda)$ stands for $I(\Lambda)=\inf \{I(\lambda): \lambda \in \Lambda\}$. Therefore, we obtain that $A_{t}^{\mu, F} / t$ converges to $C^{\prime}(0)$ exponentially as $t \rightarrow \infty$ (cf. [3]); for any $\epsilon>0$, there exist constants $k_{1}, k_{2}>0$ such that

$$
P_{i}\left(\left|\frac{A_{t}^{\mu, F}}{t}-C^{\prime}(0)\right|>\epsilon\right) \leq k_{1} e^{-k_{2} t} .
$$




\section{REFERENCES}

[1] Cinlar, E.: Introduction to Stochastic Processes, Prentice-Hall, Inc., Englewood Cliffs, N. J., 1975. MR 52:1809

[2] Dembo, A., Zeitouni, O.: Large Deviations Techniques and Applications, Second edition. Applications of Mathematics, 38. Springer-Verlag, New York, 1998. MR 99d:60030

[3] Ellis, R. S.: Large deviations for a general class of random vectors, Ann. Probab. 12 (1984), 1-12. MR 85e:60032

[4] Fitzsimmons, P. J.: On the quasi-regularity of semi-Dirichlet forms, preprint.

[5] Freedman, D.: Approximating Countable Markov Chains, Holden-Day, 1972. MR 55:1493

[6] Fukushima, M., Oshima, Y., Takeda, M.: Dirichlet Forms and Symmetric Markov Processes, de Gruyter Studies in Mathematics, 19, Walter de Gruyter \& Co., Berlin, 1994. MR 96f:60126

[7] Kim, D.: Asymptotic properties for continuous and jump type's Feynman-Kac functionals, Osaka J. Math. 37 (2000), 147-173. CMP 2000:10

[8] Kato, T.: A Short Introduction to Perturbation Theory for Linear Operators, SpringerVerlag, 1982. MR 83m:47015

[9] Sharpe, M.: General Theory of Markov Processes, Academic Press, 1988. MR 89m:60169

[10] Silverstein, M.: Symmetric Markov Processes, Springer Lect. Notes in Math. 426, 1974. MR 52:6891

[11] Takeda, M.: On a large deviation for symmetric Markov processes with finite lifetime, Stochastics and Stochastic Reports 59 (1996), 143-167. MR 98b:60057

[12] Takeda, M.: Exponential decay of life times and a theorem of Kac on total occupation times, Potential Analysis 11 (1999), 235-247. MR 2000i:60084

[13] Ying, J.: Bivariate Revuz measures and the Feyman-Kac formula, Ann. Inst. Henri Poincare, 32 (1995), 251-287. MR 97j:60139

Department of Mathematics, Pusan National University, Pusan, 609-735, Korea

Current address: Department of Systems and Information, Graduate School of Science and Technology, Kumamoto University, Kurokami, 2-39-1, Kumamoto 860-8555, Japan

E-mail address: daehong@cs.kumamoto-u.ac.jp

Mathematical Institute, Tohoku University, Sendai 980-8578, Japan

E-mail address: takeda@math.tohoku.ac.jp

Department of Mathematics, Zhejiang University, Hangzhou 310027, People's RepubLIC OF CHINA

E-mail address: jying@math.zju.edu.cn 